ISSN 2447-9071

doi https://doi.org/10.36414/rbmc.v6i15.39

Contato para correspondência: Leonardo Luiz Borges

E-mail:

leonardo.cbb@pucgoias.edu.br

Conflito de interesse: Não

Financiamento: Recursos próprios

Recebido: 16/05/2020

Aprovado: 16/06/2020

\section{Busca de moléculas com atividade broncodilatadora na espécie Mikania glomerata spreng empregando ferramentas in silico}

\section{Research for molecules with bronchodilator activity in the species Mikania glomerata spreng using in silico tools}

\author{
Letícia Fernandes Fraga', Leonardo Luiz Borges ${ }^{1,2}$ \\ 'Pontifícia Universidade Católica de Goiás - PUC Goiás \\ ${ }^{2}$ Universidade Estadual de Goiás - UEG
}

\begin{abstract}
Resumo
Esteestudo tem por objetivo avaliar os principais compostos que expliquem a atividade broncodilatadora da Mikaniaglomerata Spreng, empregando ferramentas in silico. Osmetabólitos do guaco foram levantados bibliograficamentee a codificação das moléculas para a realização das predições foiobtida no site Pubchem. Realizou-se então triagem de bioatividade com os programas SwissADME, ProToxll, PASS eMolinspiratione pesquisa de alvos, comos servidores SuperPred Webserver. Após a identificação do alvo, a estrutura selecionada foi obtida pelo site Protein Data Bank (PDB) para o docking molecular com o programa GOLD. Os metabólitos da Mikania glomerata Spreng tiveram suas propriedades físico-químicas e biológicas analisadas. Os alvos para o docking molecular foram identificados e verificados para cada composto, com suas respectivas estruturas cristalografadas no Protein Data Bank (PDB). A molécula de cumarina foi selecionada pois apresentou predição de interação com o receptor muscarínico M3 (ID: 4DAJ). O docking revelou interação da cumarina com o receptor M3, o que poderia auxiliar na explicação para os efeitos broncodilatadores desta espécie vegetal. O estudo in silico do guaco, abordado neste trabalho, elegeu a cumarina como principal metabólito ativo com possivel atividade broncodilatadora presente na Mikania glomerata Spreng. O docking da cumarina mostrou ancoragem desta molécula no sítio ativo do receptor muscarínico M3 devido as atividades desta espécie, assim, este marcador poderia atuar como antagonista desse receptor, apresentando possivel atividade parassimpatolítica e, portanto, broncodilatadora.
\end{abstract}

Palavras-Chave: Broncodilatadores, Guaco, Simulação de Acoplamento Molecular.

\section{Abstract}

This study aims to evaluate the main compounds that explain the bronchodilator activity of Mikania glomerata Spreng using in silico tools. Guaco metabolites were surveyed bibliographically and the coding of the molecules to make predictions was obtained from the Pubchem website. Bioactivity was then screened with the SwisSADME, ProToxll, PASS and Molinspiration programs and targeting with the SuperPred Webserver servers. After target identification, the selected structure was obtained from the Protein Data Bank (PDB) website for molecular docking with the GOLD program. The metabolites of Mikania glomerata Spreng had their physicochemical and biological properties analyzed. Molecular docking targets were identified and verified for each compound, with their respective structures crystallized in the Protein Data Bank (PDB). The coumarin molecule was selected because it showed prediction of interaction with the muscarinic receptor M3 (ID: $4 D A J)$. Docking revealed interaction of coumarin with the $M 3$ receptor, which could help explain the bronchodilator effects of this plantspecies. This study of guaco, using in silico tools, chose coumarin as the main active metabolite with possible bronchodilator activity present in Mikania glomerata Spreng. Coumarin docking showed anchorage 
of this molecule at the active site of the M3 muscarinic receptor due to the activities of this species, thus, this marker could act as antagonist of this receptor, presenting possible parasympatholytic and therefore bronchodilatory activity.

Keywords: Guaco, Bronchodilator Agents, Molecular Docking Simulation.

\section{Introdução}

Mikania glomerata Spreng é uma espécie pertencente à família Asteraceae, popularmente conhecida como Guaco. Nos extratos obtidos dessa espécie, já foram identificadas as seguintes classes de metabólitos secundários: cumarinas, triterpenoides, esteroides, heterosídeos, flavonoides e óleos essenciais ${ }^{1}$. Amplamente utilizado a fim de tratar sintomas como tosse, expectoração, rouquidão e dispneia, ele tem seu uso seguro reportado em diversas bases de dados, sendo considerada uma espécie de interesse do Sistema Único de Saúde (SUS)².

O Guaco faz parte da gama de plantas medicinais que são utilizadas no tratamento de diferentes condições clínicas dentro da Medicina ${ }^{3}$. Existem registros que datam do século XIX que evidenciam o uso do caule e das folhas da Mikania glomerata Spreng, espécie esta que é geograficamente distribuída em regiões tropicais e subtropicais da América, Ásia e África, sendo nativa do Brasil e cultivada em todo território nacional'.

Para que exista segurança no uso de plantas medicinais, investigações acerca das atividades biológicas e toxicológicas dos compostos identificados são necessárias. Nesse contexto, a Química Medicinal moderna apresenta destaque por ser capaz de identificar quais são as moléculas responsáveis pela atividade biológica de uma espécie investigada, além de sugerir novos candidatos a fármacos a partir desses compostos bioativos4. Recentemente, com os avanços da biologia molecular, esse processo conta com o advento da farmacologia computacional e da bioinformática que permitem realizar uma triagem de moléculas que se ligam a determinados biorreceptores. Assim, um novo candidato a fármaco pode surgir selecionando moléculas bioativas, empregando alvos moleculares e definindo vias bioquímicas que estes compostos podem interferir ${ }^{4}$. Dessa forma, utilizando-se desse planejamento é possível predizer e verificar atividades biológicas, de interesse à farmacologia, das moléculas presentes em um fármaco em potencial.

Por fim, apesar do uso difundido e do crescente interesse por seu potencial farmacológico, o xarope de guaco ainda não tem todas as suas propriedades estudadas e, principalmente, não é descrito na literatura quais compostos poderiam ser os responsáveis por suas atividades terapêuticas. Este trabalho almeja encontrar alvos moleculares que expliquem o efeito dos marcadores químicos presentes nesta espécie quanto a atividade broncodilatadora. Além disso, o screening virtual dos componentes da Mikania Glomerata Spreng, para identificar sua provável atividade broncodilatadora, através da busca por moléculas com atividade no sistema respiratório, pode servir para futuros testes in vitro e in vivo que busquem selecionar um candidato a fármaco a partir do guaco.

\section{Métodos}

Os metabólitos ativos presentes na Mikania Glomerata Spreng foram identificados através de busca de artigos científicos sobre a espécie Mikania Glomerata Spreng. Após a identificação dessas estruturas, a codificação das moléculas para a realização das predições foi obtida no site Pubchem para as análises posteriores ${ }^{5}$. A etapa de triagem de bioatividade para os marcadores químicos foi realizada com os programas SwissADME ${ }^{6}$, ProToxIII-9, PASS $^{10-12}$ e Molinspiration ${ }^{13}$. Na etapa de triagem foi possível obter uma lista com possíveis atividades biológicas para cada uma das substâncias em função de suas estruturas químicas, segundo as bases metodológicas de cada programa.

A partir dos compostos selecionados, foi realizada pesquisa de possíveis alvos para a verificação do docking molecular utilizando o programa SuperPred Webserver ${ }^{14}$, um servidor para a predição de alvos moleculares com potencial de interação com os ligantes investigados. Os alvos que apresentaram relação com a atividade biológica investigada foram obtidos no banco de dados Protein Data Bank (PDB) ${ }^{15}$. Os compostos com maior pontuação para a atividade broncodilatadora foram selecionadas para simulações de docagem molecular. O visualizador Hermes GOLD Suite 5.7.0 foi usado para preparar os receptores para o acoplamento ${ }^{16}$. A região de interesse usada para o docking foi definida como todos os resíduos de proteína dentro dos ligantes de referência que acompanhavam os complexos de proteína baixados. Valores padrão de todos os outros parâmetros foram utilizados e os complexos foram submetidos a corridas de algoritmos genéticos usando a função de adequação CHEMPLP ${ }^{17}$.

Para a validação dos parâmetros do modelo foi realizado o redocking utilizando o complexo ligante-proteína cristalografada e essas condições foram utilizadas para a realização do docking com os melhores ligante da espécie Mikania Glomerata Spreng. 


\section{Resultados}

Os principais metabólitos secundários presentes na espécie Mikania Glomerata Spreng foram: cumarina, ácido caurenoico, lupeol, ácido grandiflórico, kaurenol, beta-sitosterol, friedelina, estigmasterol, ácido cumarínico e ácido cinamoilgrandiflórico (Figura 1).

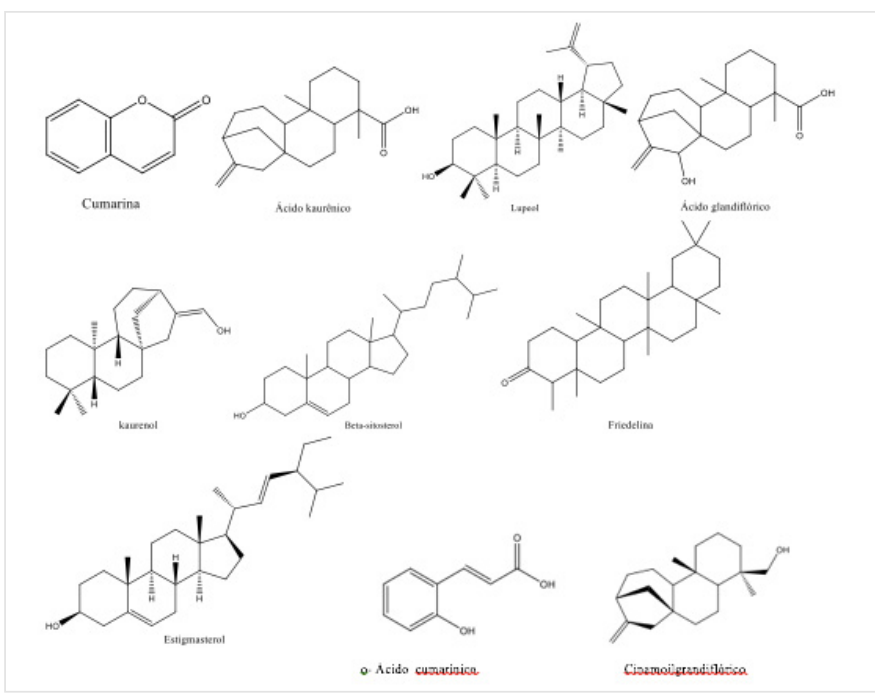

Figura 1. Estruturas presentes na Mikania Glomerata Spreng.
Cada um desses compostos foi submetido à triagem farmacocinética utilizando os programas SwissADME e Molinspiration, contendo os parâmetros: massa molecular, nOHNH, nON, TPSA, milogP, necessários para a caracterização farmacocinética dessas substâncias. Foi realizada a classificação Druglikeness nesses compostos de acordo com os critérios de Lipinski, que refletem suas respectivas capacidades de apresentarem comportamento semelhante a fármacos empregados por via oral, sendo que tais informações foram obtidas utilizando o programa SwissADME e segundo tais critérios, foi observado que todos os compostos podem ser classificados como drogas.

A capacidade de absorção pelo trato gastrointestinal (TGI), permeabilidade na barreira hematoencefálica (BHE), dose letal média (DL50) e classe de toxicidade foram obtidas nos programas SwissADME e ProToxll. Já as possíveis atividades biológicas para cada metabólito ativo da Mikania Glomerata Spreng, foram obtidas com o programa PASS. Todos esses resultados estão sintetizados na Tabela 1.

Tabela 1. Propriedades físico-químicas de cada metabólito ativo da Mikania glomerata Spreng (obtidas nos programas

\begin{tabular}{|c|c|c|c|c|c|c|c|c|c|c|c|c|}
\hline Composto & $M W$ & nOHNH & $n O N$ & TPSA & milogP & Druglikeness & Violações & $T G I$ & BHE & DL50 & $\begin{array}{l}\text { Classe de } \\
\text { toxicidade }\end{array}$ & $\begin{array}{c}\text { Atividade no siste- } \\
\text { ma respiratório }\end{array}$ \\
\hline Cumarina & 146,15 & 2 & 0 & 30,21 & 2,01 & Yes & 0 & High & Yes & $196 \mathrm{mg} / \mathrm{kg}$ & 3 & Yes \\
\hline Ácido Kaurênico & 302,46 & 1 & 2 & 37,3 & 4,67 & Yes & 1 & High & Yes & $1000 \mathrm{mg} / \mathrm{kg}$ & 5 & No \\
\hline Lupeol & 426,73 & 1 & 1 & 20,23 & 8,29 & Yes & 1 & Low & No & $2000 \mathrm{mg} / \mathrm{kg}$ & 4 & Yes \\
\hline Ácido Grandiflórico & 328,46 & 2 & 3 & 57,53 & 3,75 & Yes & 0 & High & Yes & $1000 \mathrm{mg} / \mathrm{kg}$ & 4 & No \\
\hline Kaurenol & 288,48 & 1 & 1 & 20,23 & 4,79 & Yes & 1 & High & Yes & $5000 \mathrm{mg} / \mathrm{kg}$ & 5 & No \\
\hline Beta-Sitosterol & 424,72 & 1 & 1 & 20,23 & 8,62 & Yes & 1 & Low & No & $890 \mathrm{mg} / \mathrm{kg}$ & 4 & Yes \\
\hline Friedelina & 426,73 & 0 & 1 & 17,07 & 7,85 & Yes & 1 & Low & No & $500 \mathrm{mg} / \mathrm{kg}$ & 4 & Yes \\
\hline Estigmasterol & 412,7 & 1 & 1 & 20,23 & 7,87 & Yes & 1 & Low & No & $890 \mathrm{mg} / \mathrm{kg}$ & 4 & Yes \\
\hline o- Ácido Cumarínico & 164,16 & 2 & 3 & 57,23 & 1,67 & Yes & 0 & High & Yes & $2850 \mathrm{mg} / \mathrm{kg}$ & 5 & Yes \\
\hline $\begin{array}{l}\text { Cinamoilgrandi- } \\
\text { flórico }\end{array}$ & 288,48 & 1 & 1 & 20,23 & 4,79 & Yes & 1 & High & Yes & $5000 \mathrm{mg} / \mathrm{kg}$ & 5 & Yes \\
\hline
\end{tabular}

Molinspiration e Swiss ADME); classificação druglikeness segundo a Regra dos Cinco de Lipinski, e seus números de violações (obtidos no software SwissADME); capacidade de

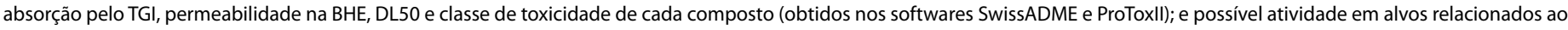
sistema respiratórios prevista para cada composto (segundo o programa PASS). 
Na pesquisa de alvos para a realização do docking molecular utilizando o programa SuperPred Webserver foram verificados os alvos de cada composto e suas respectivas estruturas cristalografadas no PDB (Protein Data Bank). Dos compostos apresentados na Tabela 1, a molécula de cumarina foi selecionada por apresentar potencial interação com o receptor muscarínico $\mathrm{M} 3$, que pode estar associado à broncodilatação, pois seu bloqueio acarretaria efeito parassimpatolítico. A estrutura ID: 4DAJ foi a utilizada para a realização do estudo de ancoragem molecular com o ligante selecionado ${ }^{18}$.

O ligante selecionado por este estudo foi a cumarina pois foi ela o metabólito secundário do Guaco que teve melhor desempenho e maior potencial de interação com o receptor muscarínico M3 nas análises feitas por este trabalho. A cumarina foi classificada como druglikeness segundo os critérios de Lipinski, sendo portanto uma droga em potencial; apresentou alta absorção pelo trato gastrointestinal (TGI) e pela barreira hematoencefálica (BHE); com menor dose letal média (DL50) e menor classe de toxicidade entre todos os compostos analisados; além de que mostrou possível atividade em alvos do sistema respiratório, segundo o programa PASS.

Realizou-se inicialmente o redocking (Figura 2) para a validação dos parâmetros do modelo, utilizando o ligante cristalografado tiotrópio e como sítio de ligação a estrutura 4DAJ a fim de demonstrar a ocorrência da ligação do tiotrópio na estrutura do receptor muscarínico M3, na mesma posição da estrutura depositada no PDB. Após a definição dos parâmetros do modelo a ser empregado para o docking, foram realizadas simulações com a cumarina, sobre o sítio ativo do alvo 4DAJ ${ }^{18}$.

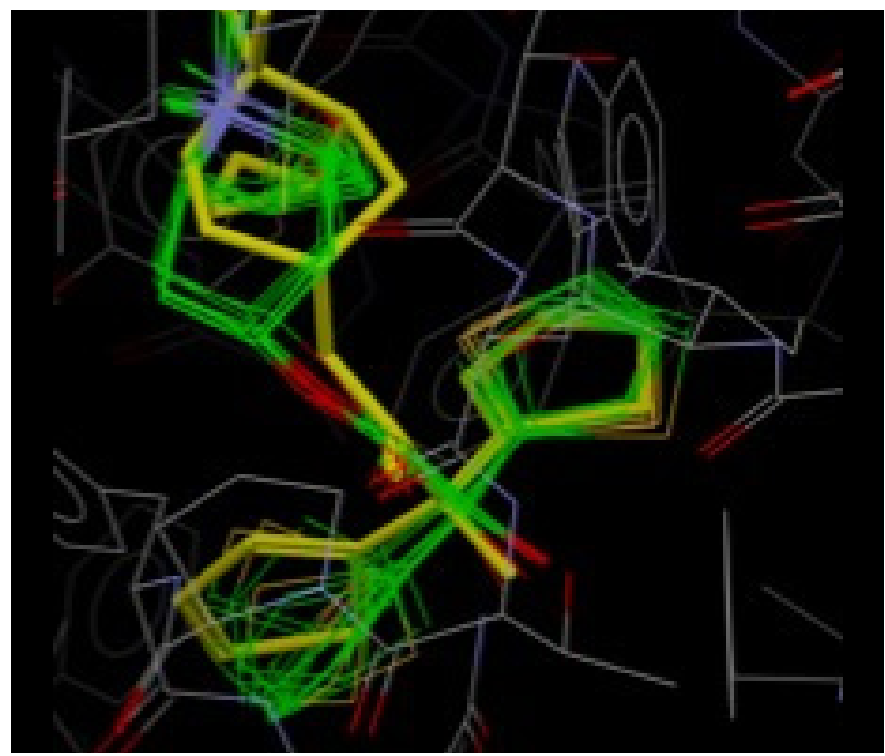

Figura 2. Redocking do tiotrópio no receptor muscarínico M3 (os valores de RMSD das 10 poses geradas foram próximos a 1).
O docking revela uma interação da cumarina com o receptor muscarínico M3. A Figura 3 revela que uma interação intermolecular de maior intensidade (ligação de hidrogênio) ocorre entre a cumarina e o aminoácido serina 518 presente na cavidade ativa do receptor muscarínico M3. Outras interações provavelmente responsáveis pela estabilização do ligante no sítio ativo correspondem a interações do tipo $\pi-\pi$ com os aminoácidos tirosina 515, 832, 809 e com o resíduo de cisteína 835. A conformação tridimensional da interação da cumarina com o receptor muscarínico M3 está representada na Figura 4.

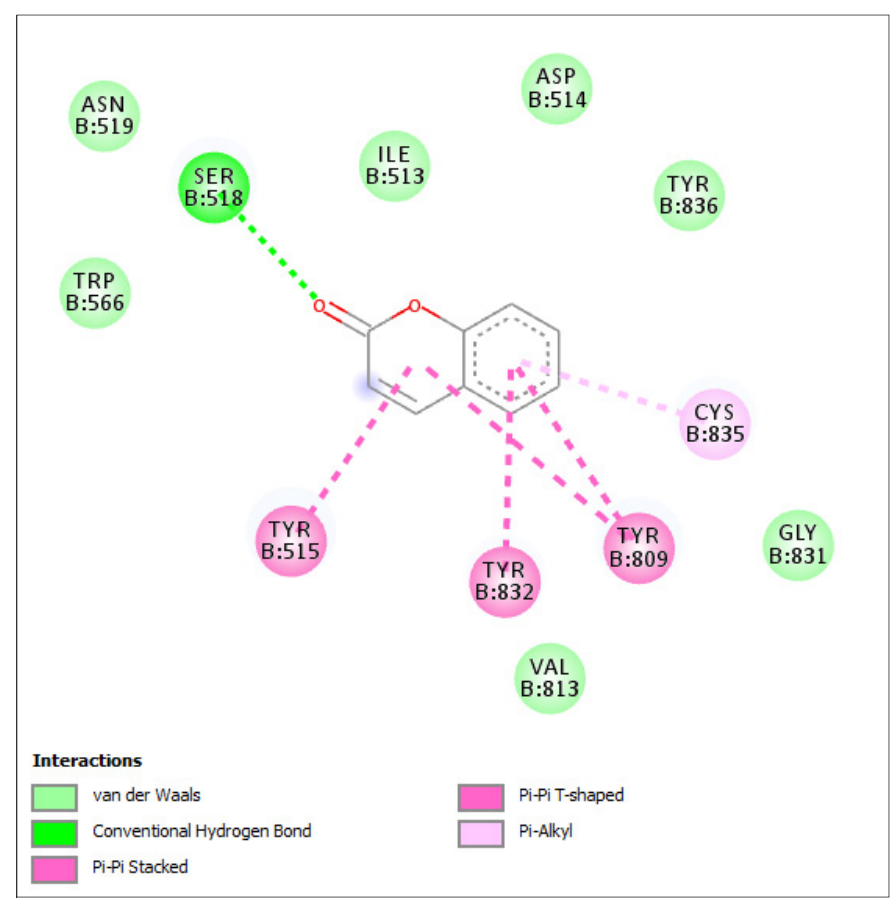

Figura 3. Pose 1 da ancoragem da cumarina dentro do sítio do receptor muscarínico. Figura gerada com o software Pymol 1.1r1 software.

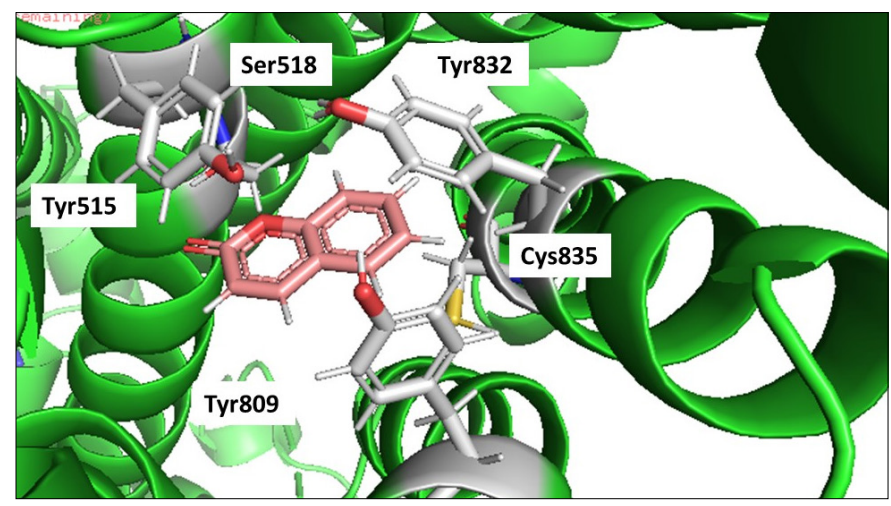

Figura 4. Diagrama 2D de interação da cumarina posição 1 no receptor muscarínico M3. Essa figura foi gerada com Discovery Studio 3.5 Visualizer. 


\section{Discussão}

O levantamento de metabólitos ativos da Mikania Glomerata Spreng, com suas respectivas propriedades físico-químicas, classificação Druglikeness, predição de toxicidade, predição de possível ação no sistema respiratório, além da análise dos alvos para realização do docking permitiu a escolha da cumarina como molécula para realização do docking molecular, apresentando melhor desempenho em todas essas análises, com maior potencial de interação com o receptor muscarínico M3.

Todos os com postos elencados na triagem realizada por este estudo puderam ser classificados como drogas, segundo Lipinski 1997. Essa classificação é amplamente utilizada para determinar propriedades moleculares que são importantes para a predição farmacocinética das substâncias in vivo. De acordo com a regra dos cinco de Lipinski, em que se baseia essa classificação, uma molécula candidata é mais provável de possuir propriedades favoráveis se o peso molecular for abaixo de 500 dáltons, o coeficiente de partição octanol/água (log P) é inferior 5 , se não há mais que 5 doadores de ligação de hidrogênio (grupos $\mathrm{OH}$ e $\mathrm{NH}$ ) e se não existem 10 aceptores de ligação de hidrogênio (nomeadamente $\mathrm{N}$ and $\mathrm{O})^{19}$.

A cumarina se comportou neste estudo in silico, como um ligante na estrutura do receptor muscarínico M3 (4DAJ), permitindo a inferência da possível ação parassimpatolítica dessa molécula, de forma semelhante ao que foi demonstrado no redocking do tiotrópio, substância broncodilatadora com ação anticolinérgica, que se ligou à mesma estrutura do receptor muscarínico $\mathrm{M} 3$, revelando possível semelhança portanto com a cumarina.

A cumarina ocupou o sítio ativo da acetilcolina, possivelmente funcionando, portanto, como um antagonista competitivo da acetilcolina e atuando de forma semelhante ao tiotrópio, o que poderia colaborar para os efeitos broncodilatadores do extrato da espécie Mikania Glomerata Spreng ${ }^{20,21}$. A Farmacopeia Brasileira quarta edição, 2005, considera a cumarina (1,2-benzopirona) o marcador químico da espécie Mikania Glomerata Spreng, reportado como composto que apresenta atividade anti-inflamatória, o que poderia favorecer os efeitos benéficos sobre a asma. A cumarina foi descrita como capaz de reduzir significativamente $(p<0,01)$ a infiltração celular na cavidade pleural de ratos (concentração de $75 \mathrm{mg} / \mathrm{kg}$ )22, e também já foi relatado que a cumarina foi capaz de atenuar a resposta inflamatória, diminuindo a infiltração tecidual leucocitária, na pleurisia induzida por carragenana in ratos ${ }^{23,24}$.

Além dos efeitos anti-inflamatórios, existem evidências que sugerem que a cumarina é capaz de promover o relaxamento da musculatura lisa em modelos de traqueia isoladas de cobaia25 e um bloqueio dos canais de cálcio. Os modelos aqui descritos sugerem que um mecanismo anticolinérgico poderia corroborar para os efeitos deste marcador da espécie Mikania Glomerata Spreng.

Levantamentos de várias espécies vegetais que apresentam cumarina mostraram que essas espécies compartilhavam além do efeito broncodilatador, também efeitos anti-inflamatório e antinociceptivo ${ }^{26}$.

Por fim, a espécie Mikania Glomerata Spreng, o popular Guaco, tem sido utilizada de maneira crescente no Brasil com a finalidade de tratar condições clínicas das vias aéreas, principalmente a dispneia, sendo de interesse do Sistema único de Saúde (SUS). Seu estudo in silico, abordado neste trabalho, elencou a cumarina como principal metabólito ativo com possível atividade broncodilatadora presente na Mikania glomerata Spreng. A molécula de cumarina foi selecionada para a realização de docking molecular pois apresentou maior predição de interação com o receptor muscarínico M3 (identificado pelo PDB como estrutura 4DAJ), se ancorando no sítio ativo desse receptor, e foi considerada pelas análises farmacológicas computacionais descritas neste artigo, como um antagonista competitivo desse receptor, atuando como inibidora da acetilcolina e apresentando portanto uma possível atividade parassimpatolítica e portanto broncodilatadora, a despeito de sua atividade anti-inflamatória já anteriormente constatada.

Dessa forma, o presente artigo, ao se utilizar de técnicas in silico com a Mikania Glomerata Spreng, levantou dados importantes para o planejamento futuro de um possível fármaco que utilize essa espécie vegetal como base. Logo, é imprescindível que ocorra a realização de novos trabalhos desenvolvidos in vitro e in vivo para aprofundar o estudo do Guaco como um fármaco broncodilatador.

\section{Conclusão}

O estudo in silico do guaco, abordado neste trabalho, elegeu a cumarina como principal metabólito ativo com possível atividade broncodilatadora presente na Mikania glomerata Spreng. O docking da cumarina mostrou ancoragem desta molécula no sítio ativo do receptor muscarínico M3 devido as atividades desta espécie, assim, este marcador poderia atuar como antagonista desse receptor, apresentando possível atividade parassimpatolítica e, portanto, broncodilatadora.

\section{Referências}

1. Brasil. Ministério da Saúde - Secretaria de Ciência, Tecnologia e Insumos Estratégicos Departamento de Assistência Farmacêutica e Insumos Estratégicos. Guaco - Mikania glomerata Spreng., Asteraceae. Informações Sistematizadas da Relação Nacional de Plantas 
Medicinais de interesse ao SUS. 2018;1-92.

2. Silva LS, Silva LS, Brumano L, Stringheta PC, Pinto MAO, Dias LOM, et al. Preparation of dry extract of Mikania glomerata Sprengel (Guaco) and determination of its spectrophotometry and HPLC-UV. Molecules. 2012;17(9):10344-54.

3. Czelusniak KE, Brocco A, Pereira DF, Freitas GBL. Farmacobotânica, fitoquímica e farmacologia do Guaco: revisão considerando Mikania glomerata Sprengel e Mikania laevigata Schulyz Bip. ex Baker. Revista Brasileira de Plantas Medicinais. 2012;14(2):400-09.

4. Lima LM. Química medicinal moderna: desafios e contribuição brasileira. Química Nova. 2007;30(6):1456-68.

5. Kim S, Chen J, Cheng T, Gindulyte A, He J, He S, et al. PubChem 2019 update: improved access to chemical data. Nucleic Acids Research. 2019;47(D1):D1102-09. Avaiable from: https://pubchem.ncbi.nlm.nih.gov/

6. Daina A, Michielin O, Zoete V. SwissADME: a free web tool to evaluate pharmacokinetics, drug-likeness and medicinal chemistry friendliness of small molecules. Scientific Reports. 2017;7(42717).

7. Banerjee P, Eckert AO, Schrey AK, Preissner R. ProTox-II: a webserver for the prediction of toxicity of chemicals. Nucleic Acids Research. 2018;46(2):W257-W263.

8. Banerjee P, Dehnbostel FO, Preissner R. ProTox-II: a webserver for the prediction of toxicity of chemicals. Frontiers in Chemistry. 2018;6(362).

9. Drwal MN, Banerjee P, Dunkel M, Wettig MR, Preissner R. ProTox: a web server for the in silico prediction of rodent oral toxicity. Nucleic Acids Research. 2014;42W: 53-8.

10. Poroikov VV, Filimonov DA. How to acquire new biological activities in old compounds by computer prediction. Journal of Computer-Aided Molecular Design. 2002;16(11):819-24.

11. Poroikov VV, Filimonov DA, Borodina YV, Lagunin AA, Kos A. Robustness of Biological Activity Spectra Predicting by Computer Program PASS for Noncongeneric Sets of Chemical Compounds. Journal of Chemical Information and Computer Scientists. 2000;40(6):134955.

12. Sadym A, Lagunin A, Filimonov D, Poroikov V. Prediction of Biological Activity Spectra via The Internet. Journal SAR and QSAR in Environmental Research. 2003;14(5-6):339-47.

13. Jarrahpour A, Fathi J, Mimouni M, Hadda T, Sheikh J, Chohan Z, et al. Petra, Osiris and Molinspiration (POM) Together as a Successful Support in Drug Design: An- tibacterial Activity and Biopharmaceutical Characterization of Some Azo Schiff Bases. Medicinal Chemistry Research. 2012;21(8):1984-90.

14. Nickel J, Gohlke BO, Erehman J, Banerjee P, Rong WW, Goede A, et al. SuperPred: update on drug classification and target prediction. Nucleic Acid Res. 2014;42W:26-31.

15. Berman HM, Westbrook J, Feng Z, Gilliland G, Bhat TN, Weissig $\mathrm{H}$, et al. The Protein Data Bank. Nucleic Acids Research. 2000;28(1):235-42. Avaiable from: https:// www.rcsb.org/

16. Cole JC, Nissink JWM, Taylor R. Protein-ligand Docking and Virtual Screening with GOLD in Virtual Screening in Drug Discovery; Shoichet B, Alvarez J, Eds.; Taylor \& Francis. CRC Press. 2005.

17. Korb O, Stützle T, ExnerTE. Empirical scoring functions for advanced protein-ligand docking with PLANTS. Journal of Chemical Information and Modeling. 2009;49(1):84-96.

18. Kruse $A C, H u J$, Pan $A C$, Arlow DH, Rosenbaum $D M$, Rosemond $E$, et al. Structure and dynamics of the M3 muscarinic acetylcholine receptor. Nature. 2012;482(7386): 552-6.

19. Lipinski CA, Lombardo F, Dominy BW, Feeney PJ. Experimental and computational approaches to estimate solubility and permeability in drug discovery and development settings. Advanced Drug Delivery Reviews. 1997;23: 4-25.

20. Alvarenga FCR., Garcia EF, Bastos EMAF, Grandi TSM, Duarte MGR. Evaluation of the quality of commercial samples of leaves and tinctures of guaco. Brazilian Journal of Pharmacognosy. 2009;19: 442-8.

21. Brasil. Farmacopeia Brasileira. Fourth ed., Part II, 6th fasc ed. São Paulo: Atheneu; 2005.

22. Della Pasqua CSP, Iwamoto RD, Antunes E, Borghi AA, Sawaya ACFH, Landucci ECT. Pharmacological Study of Anti-inflammatory Activity of Aqueous Extracts of Mikania glomerata (Spreng.) and Mikania laevigata (Sch. Bip. Ex Baker). Journal of Ethnopharmacology. 2018.

23. Zhi-Peng $L$, Jin-Feng $H$, Ming-Na $S$, Hai-Jie J, Ming $Z$, Dong-Hui W, et al. Effect of compound IMMLG5521, a novel coumarin derivative, on carrageenan-induced pleurisy in rats. European Journal of Pharmacology. 2011; 661: 118-23.

24. Fierro IM, Silva ACB, Lopes CS, Moura RS, Barja-Fidalgo C. Studies on the anti-allergic activity of Mikania glomerata. Journal of Ethnopharmacology. 1999;66: 19-24.

25. Leite MGR, Souza CL, Silva MAM, Moreira LKA, Matos 
FJA, Viana GSB. Estudo farmacológico comparativo de Mikania glomerata Spreng (guaco), Justicia pectoralis Jacq (anador) e Torresea cearensis Fr. All (cumarú). Revista Brasileira de Farmácia. 1993; 1:12-15.

26. Leal LK, Ferreira AA, Bezerra GA, Matos FJ, Viana GS. Antinociceptive, anti-inflammatory and bronchodilator activities of Brazilian medicinal plants containing coumarin: a comparative study. Journal of Ethnopharmacology. 2000;70(2):151-9. 